\title{
The second spectrum of Timoshenko beam theory-Further assessment
}

\author{
N.G. Stephen* \\ School of Engineering Sciences, Mechanical Engineering, University of Southampton, \\ Highfield, Southampton SO17 1BJ, UK
}

Received 21 March 2005; received in revised form 1 August 2005; accepted 2 August 2005

Available online 5 October 2005

\begin{abstract}
A review of contributions and views on the second spectrum of Timoshenko beam theory (TBT) over the past two decades, together with some new results, are presented. It is shown that the Timoshenko frequency equation factorises not solely for hinged-hinged end conditions, as is often claimed, but also for guided-guided and guided-hinged; these new cases may be regarded as portions of a multi-span hinged-hinged beam. A higher-derivative Lagrangian that leads directly to the well-known fourth-order Timoshenko beam equation is reviewed. A simple relationship between the so-called Ostrogradski energy and the mechanical energy is derived for hinged-hinged end conditions. It is shown that the Ostrogradski energy is positive for the first spectrum but negative for the second; within some branches of physics, this would be sufficient to conclude that the second spectrum is "unphysical". A numerical example presented by Levinson and Cooke is re-examined using both TBT and exact plane stress elastodynamic theory. Agreement is excellent for the first spectrum. However, the second spectrum predictions are not in consistent agreement with any single mode of vibration. For long wavelength it is very close to the second asymmetric mode, but as wavelength shortens, it becomes closer to the second symmetric, then the third asymmetric modes. The conclusion remains unchanged: the second spectrum predictions of TBT should be disregarded.

(C) 2005 Elsevier Ltd. All rights reserved.
\end{abstract}

\footnotetext{
*Tel. + 442380592359 ; fax: + 442380593230 .

E-mail address: ngs@soton.ac.uk.
} 


\section{Introduction}

The possibility of a so-called second spectrum of natural frequency predictions from Timoshenko beam theory (TBT) was first raised by Traill-Nash and Collar [1] in 1953. Since that time, two issues have attracted considerable research interest: the first is the validity of the second spectrum frequency predictions, while the second is the existence of the second spectrum for beam end conditions other than hinged-hinged. The present author concluded in 1982 [2] that the second spectrum of TBT is "an inevitable but meaningless consequence of the structure of an otherwise excellent approximate theory." The basis of that conclusion was a comparison between the phase velocity predictions from the exact (according to the spirit of the mathematical theory of elasticity) Pochhammer-Chree (PC) theory for an infinite rod of circular cross-section, and the equivalent predictions from TBT. Excellent agreement was found for the lowest flexural mode of vibration over a wide range of wavelength. However, no consistent agreement could be found for the second spectrum (TBT2) predictions; at long wavelength the TBT2 prediction was close to the second flexural mode of PC theory, as one would wish, but at shorter wavelength TBT2 agrees more closely with the second longitudinal mode of PC theory.

In a similar analysis [3], the author has also considered the validity of the higher spectra predictions of Mindlin plate theory, and concluded that the so-called $w_{2}$-mode should be disregarded, again since consistent agreement with exact predictions from Rayleigh-Lamb plate theory could not be found; on the other hand, the lowest $w_{1}$-spectrum has best agreement when one employs the shear coefficient $\kappa=5 /(6-v)$, and the so-called $H$-mode is in exact agreement for $\kappa=\pi^{2} / 12$.

Here, the comparison made in Ref. [2] for wave propagation in an infinite beam of circular cross-section, is extended to standing waves in a thin rectangular (plane stress) beam of finite length, having hinged-hinged end conditions; the example chosen is that considered by Levinson and Cooke [4]. For the first spectrum, it is seen that agreement between the plane stress elastodynamic prediction and TBT1 is better than $-0.4 \%$ and $+0.55 \%$ for the first 20 natural frequencies, when one uses the shear coefficient $\kappa=5(1+v) /(6+5 v)$. In contrast, the second spectrum predictions are not in consistent agreement with any one mode; the first two natural frequencies, and the cut-off frequency, are in good agreement with the second asymmetric predictions from the plane stress theory, but the higher modes are in better agreement with the second symmetric, and then the third asymmetric predictions. Once again, it is concluded that the second spectrum frequencies should be disregarded.

The second issue, whether the second spectrum exists for beams having other than hinged-hinged end conditions, is described by Levinson and Cooke [4], and further discussed by Prathap [5]. Levinson and Cooke, along with many other researchers, were of the view that a second spectrum could exist only for hinged-hinged ends, when the frequency equation factorises, but regarded the two spectrum terminology as "tenuous" and, "at best, an aid to computation". Finite element simulations have produced conflicting conclusions: according to Bhashyam and Prathap [6] "for end conditions other than hinged-hinged, with a finite element procedure one can detect two kinds of spectra and can suitably classify them." On the other hand, Abbas and Thomas [7] only found evidence of a second spectrum of frequencies for a hinged-hinged beam.

The present author has not, until now, entered that particular debate, but has always taken the view that if, above some cut-off frequency, there are new modes of vibration whose character 
differs in some way from what one is familiar with below this cut-off (the first spectrum) then it is entirely legitimate to describe the new modes as a second spectrum, irrespective of whether the frequency equation factorises. Further, one would then wish to identify these new frequencies so that they may be disregarded, in the light of the conclusion in Ref. [2].

In 1999, Han et al. [8] provided a brief discussion on the second frequency spectrum as part of a wider study of beam theories in general, and claimed that the bending moment and shearing force are in phase for the first spectrum, but out of phase for the second. This conclusion may be erroneous, as the authors employ the expression $M=E I \psi$, rather than $M=E I \partial \psi / \partial x$, for the bending moment. Nevertheless, if true, this would be an example of character difference, as alluded to above. Despite noting that "the two pairs of modes, are indeed distinct and correspond to distinct natural frequencies", Han et al. state that there is "no need to refer to them as separate frequency spectra" and go on to quote Levinson and Cooke: "... the two spectra interpretation of the predictions of TBT is rather a matter of taste and not even a particularly fruitful interpretation at that." An earlier observation of character difference was made by Downs [9], who stated that "deformations due to shear and bending are of the same phase and are summed to give the total transverse deflection" for the first spectrum, but "the shear and bending deformation are opposed with the net transverse deflection equal to their difference" for the second. In Section 2, it is shown (and one suspects Han et al. intended to claim) that the ratio of cross-sectional rotation to shear angle $(\psi / \gamma)$ is of a different sign for the two spectra, at least for hinged-hinged end conditions. This character difference was first noted by Traill-Nash and Collar [1].

In a 2001 contribution, Ekwaro-Osire et al. [10] provided a scheme which facilitates a progressive ordering of all of the natural frequencies, and their mode shapes, and claimed that this "eliminates the remaining argument for the two spectra interpretation", and "puts to rest the last remaining criticism of the single-spectrum interpretation of the Timoshenko beam model structure."

The present author remains surprised that so many researchers should favour this singlespectrum interpretation: for problems in wave propagation, a frequency-wavenumber (dispersion) diagram will show several branches for each of the family of waves for which the nature of the lowest branch (for example, flexural, torsional or extensional-or, more technically, asymmetric, torsional axisymmetric and torsionless axisymmetric, respectively) is often adopted as the descriptor. Thus Miklowitz [11] writes "The basic advantages of the Timoshenko theory over the Bernoulli-Euler are (1) it has closer agreement with the exact over a greater domain of the lowest branch, and (2) it has a second branch (flexural thickness-shear) which compares very favourably with the exact theory, at and near cut-off and over the imaginary loop". It seems quite natural to transfer the concept of a first and second branch for wave propagation, to a first and second spectrum when considering a standing wave problem.

Here, it is shown that the frequency equation also factorises for guided-guided (or sliding-sliding, or roller skate according to Miklowitz [11]) and guided-hinged end conditions. Factorisation occurs because the mode shapes are (co)sinusoidal, so the standing wave may be regarded as the superposition of forward and backward travelling waves within a beam of infinite length; the boundary conditions do not induce evanescent waves. As will be seen, beams having both guided-guided and guided-hinged conditions can be regarded as portions of a multiple span hinged-hinged beam. Applying wave-train phase closure principles, Wang and So [12] have noted the special nature of these combinations of end conditions, and describe the waves as degenerate. 
A third issue, related to the validity of the second spectrum, has arisen from the seemingly quite unrelated areas of field and particle physics. Although the Timoshenko beam equation is, most often, presented as a partial differential equation (PDE) that has fourth-order derivatives in both space and time, it is usually derived through application of Newton's second law for transverse and rotational acceleration, with variables $v$ and $\psi$ as transverse displacement and cross-sectional rotation, respectively, leading to two coupled PDEs containing second-order derivatives in time. These coupled equations may also be determined using Hamilton's principle by variation of the Lagrangian function $L=T-V$, where $T$ and $V$ are the kinetic and strain energies, respectively. The Lagrangian function contains only first derivatives in time, for example velocity squared. In the absence of damping, the Hamiltonian function, $H=T+V$, equal to the total mechanical energy, is an integral of the motion, that is, a conserved quantity. More often than not, one of the variables, say cross-sectional rotation $\psi$, is then eliminated in favour of transverse displacement $v$, leading to the familiar PDE of fourth order in both space and time. Chervyakov and Nesterenko [13,14] have shown that this fourth-order equation can also be found by variation of an appropriate Lagrangian function which now contains higher time derivatives. The Hamiltonian function may be constructed employing methods developed by Ostrogradski (see Ref. [15]) specifically for higher-derivative field theories, and again this Ostrogradski Hamiltonian is a conserved quantity, in some way equivalent to energy, although its precise meaning is unclear.

Within the field and particle physics community, it is understood that such higher time derivative theories can, according to Simon [16], "lead to undesirable properties", and may arise through quantum corrections to general relativity, or rigidity correction to string theory. TBT includes the second (shear deformation) correction to Euler-Bernoulli beam theory, the first being the less important rotary inertia correction introduced by Rayleigh. This is entirely consistent with Simon's statement [16]: "Quite often the higher-derivative terms are added to a more standard (lower-derivative) theory as a correction." On the other hand [16], "There is a large class of theories naturally containing higher derivatives that do not suffer the above problems", the problems being runaway solutions, "qualitatively quite different from those of a related lowerderivative theory", and "ghost fields"; within classical continuum mechanics, coupled tension-torsion vibration provides an example of a higher-order derivative theory not beset with "undesirable properties".

In two 1993 papers, Chervyakov and Nesterenko [13,14] considered the formulation of TBT as a possible way of avoiding some of these issues; from Ref. [13]: "Here there arises a very typical picture for higher-derivative theories: in addition to the basic mode of oscillations $\cdots \cdots$ there emerge additional, as a rule, higher-frequency modes. The contribution to the energy of the second mode has the opposite sign as compared with the basic one." These negative contributions to the so-called Ostrogradski energy, $E_{O}$, are regarded as physically unacceptable; on the other hand, the structure of TBT may be useful in the interpretation of higher time derivative theories, as it has the virtues of a well-defined positive definite mechanical energy.

In Ref. [14], Nesterenko noted that "the quantity $E_{O}$ is not the same as the mechanical energy of the Timoshenko beam and its precise physical meaning is still unclear", indicating a need for further research. However, Nesterenko's views on the second spectrum were perfectly clear: "in the Timoshenko theory it is natural to regard as physical only those frequencies which turn into 
frequencies of the Bernoulli-Euler equation when the coefficients

$$
a_{2}=\frac{I}{A}\left(1+\frac{E}{\kappa G}\right) \quad \text { and } \quad a_{3}=\frac{\rho I}{\kappa A G}
$$

vanish", indicating that only the first spectrum satisfies this natural condition, and is "the only region where the theory under consideration is applicable." In contrast, the second spectrum frequencies "tend to infinity as $a_{3} \rightarrow \infty$." In Section 3, a simple relationship between the mechanical and Ostrogradski energies for TBT is derived, and it is shown that $E_{O}$ is always positive for the first spectrum, and always negative for the second.

Last, the numerical example discussed by Levinson and Cooke is re-considered using both TBT and exact plane stress theory; the latter is developed within the appendix. Agreement between the frequency predictions is excellent for the first spectrum; however, the second spectrum prediction of TBT does not provide consistent agreement with any single mode of vibration. Overall, the conclusion remains the same: the predictions of TBT2 should be disregarded.

\section{Timoshenko beam theory, and factorisation of the frequency equation}

Following the notation and sign conventions of Levinson and Cooke [4], with the exception that the shear coefficient is written as $\kappa$ rather than $\kappa^{2}$, and the transverse displacement is written as $v$ rather than $w$, the coupled equations of free vibration of a uniform Timoshenko beam are

$$
\kappa A G \frac{\partial}{\partial x}\left(\psi+\frac{\partial v}{\partial x}\right)=\rho A \frac{\partial^{2} v}{\partial t^{2}}, \quad \kappa A G\left(\psi+\frac{\partial v}{\partial x}\right)-E I \frac{\partial^{2} \psi}{\partial x^{2}}=-\rho I \frac{\partial^{2} \psi}{\partial t^{2}} .
$$

Elimination of the cross-sectional rotation $\psi$ leads to the single differential equation of the fourth order in both space and time

$$
E I \frac{\partial^{4} v}{\partial x^{4}}+\rho A \frac{\partial^{2} v}{\partial t^{2}}-\rho I\left(1+\frac{E}{\kappa G}\right) \frac{\partial^{4} v}{\partial x^{2} \partial t^{2}}+\frac{\rho^{2} I}{\kappa G} \frac{\partial^{4} v}{\partial t^{4}}=0
$$

alternatively, elimination of $v$ leads to an identical equation in $\psi$. Assuming $v(x, t)=V(x) \mathrm{e}^{\mathrm{i} \omega t}$, $\psi(x, t)=\Psi(x) \mathrm{e}^{\mathrm{i} \omega t}$, then one has

$$
\begin{aligned}
& V(x)=B \sinh \lambda_{1} x+C \cosh \lambda_{1} x+D \sin \lambda_{2} x+F \cos \lambda_{2} x, \\
& \Psi(x)=-\left(\alpha_{1} / \lambda_{1}\right)\left(B \cosh \lambda_{1} x+C \sinh \lambda_{1} x\right)+\left(\alpha_{2} / \lambda_{2}\right)\left(D \cos \lambda_{2} x-F \sin \lambda_{2} x\right),
\end{aligned}
$$

where

$$
\begin{aligned}
& \alpha_{1,2}=\left(\frac{\rho \omega^{2}}{\kappa G} \pm \lambda_{1,2}^{2}\right), \quad \lambda_{1,2}^{2}=\left(\frac{b^{4} \omega^{4}}{4}+a \omega^{2}\right)^{1 / 2} \mp \frac{b^{2} \omega^{2}}{2} \\
& a=\frac{\rho}{E}\left(\frac{A}{I}-\frac{\rho \omega^{2}}{\kappa G}\right), \quad b^{2}=\frac{\rho}{E}\left(1+\frac{E}{\kappa G}\right)
\end{aligned}
$$

and $B, C, D$ and $F$ are arbitrary constants. 
At a guided end, both the shearing force $Q=\kappa A G(\psi+\partial v / \partial x)$, and cross-sectional rotation $\psi$ are equal to zero; applying these conditions at $x=0$ and $L$ leads to the matrix equation

$$
\begin{aligned}
& {\left[\begin{array}{cccc}
-\alpha_{1} / \lambda_{1} & 0 & \alpha_{2} / \lambda_{2} & 0 \\
\left(\lambda_{1}-\alpha_{1} / \lambda_{1}\right) & 0 & \left(\lambda_{2}+\alpha_{2} / \lambda_{2}\right) & 0 \\
-\left(\alpha_{1} / \lambda_{1}\right) \cosh \lambda_{1} L & -\left(\alpha_{1} / \lambda_{1}\right) \sinh \lambda_{1} L & \left(\alpha_{2} / \lambda_{2}\right) \cos \lambda_{2} L & -\left(\alpha_{2} / \lambda_{2}\right) \sin \lambda_{2} L \\
\left(\lambda_{1}-\alpha_{1} / \lambda_{1}\right) \cosh \lambda_{1} L & \left(\lambda_{1}-\alpha_{1} / \lambda_{1}\right) \sinh \lambda_{1} L & \left(\lambda_{2}+\alpha_{2} / \lambda_{2}\right) \cos \lambda_{2} L & -\left(\lambda_{2}+\alpha_{2} / \lambda_{2}\right) \sin \lambda_{2} L
\end{array}\right]} \\
& \times\left[\begin{array}{l}
B \\
C \\
D \\
F
\end{array}\right]=\mathbf{0}
\end{aligned}
$$

and for a non-trivial solution, a zero value of the determinant leads to the frequency equation

$$
\frac{\left(\alpha_{1} \lambda_{2}^{2}+\alpha_{2} \lambda_{1}^{2}\right)^{2}}{\lambda_{1}^{2} \lambda_{2}^{2}} \sin \lambda_{2} L \sinh \lambda_{1} L=0
$$

For guided-hinged conditions, for which one now has $v=0$ and $\partial \psi / \partial x=0$ at $x=L$, the matrix equation becomes

$$
\left[\begin{array}{cccc}
-\alpha_{1} / \lambda_{1} & 0 & \alpha_{2} / \lambda_{2} & 0 \\
\left(\lambda_{1}-\alpha_{1} / \lambda_{1}\right) & 0 & \left(\lambda_{2}+\alpha_{2} / \lambda_{2}\right) & 0 \\
\sinh \lambda_{1} L & \cosh \lambda_{1} L & \sin \lambda_{2} L & \cos \lambda_{2} L \\
-\alpha_{1} \sinh \lambda_{1} L & -\alpha_{1} \cosh \lambda_{1} L & -\alpha_{2} \sin \lambda_{2} L & -\alpha_{2} \cos \lambda_{2} L
\end{array}\right]\left[\begin{array}{c}
B \\
C \\
D \\
F
\end{array}\right]=\mathbf{0}
$$

leading to the frequency equation

$$
\left(\alpha_{1}-\alpha_{2}\right) \frac{\left(\alpha_{1} \lambda_{2}^{2}+\alpha_{2} \lambda_{1}^{2}\right)}{\lambda_{1} \lambda_{2}} \cos \lambda_{2} L \cosh \lambda_{1} L=0 .
$$

An equivalent process for the hinged-hinged beam, for which the boundary conditions are $v=0$ and $\partial \psi / \partial x=0$ at $x=0$ and $x=L$, leads to

$$
\left(\alpha_{1}-\alpha_{2}\right)^{2} \sin \lambda_{2} L \sinh \lambda_{1} L=0 .
$$

Thus, we see that factorisation of the frequency equation is not confined to hinged-hinged end conditions, as is the conventional wisdom, although these new cases are related. Fig. 1 shows a three span hinged-hinged beam, for which the frequency equation would be the same as that of a single span. The addition of mass-less guides at the mid-point of each span would not affect the system, as the required conditions at a guide are guaranteed by symmetry. Portions AB and AC represent the first and second modes for the guided-guided case; higher modes of vibration are accommodated by increasing the number of spans. For the guided-hinged case, the first mode is represented by portion A2 of the system; the change from trigonometric and hyperbolic sine functions in the frequency Eq. (6) to equivalent cosine functions within the frequency Eq. (8), is the consequence of a change in the effective length of the beam, $L_{\text {eff }}$, according to the scheme 


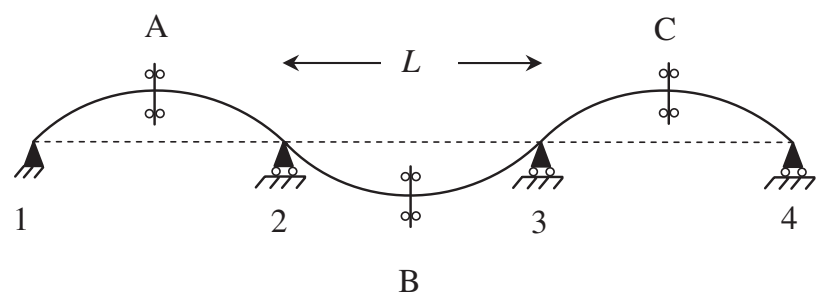

Fig. 1. Three-span hinged-hinged beam with guides at mid-span. Portions AB and AC represent the first and second modes, respectively, for guided-guided end conditions. Portions A2 and A3 represent the first and second modes for guided-hinged.

$L_{\mathrm{eff}}=(2 n-1) L /(2 n)$. Thus for $n=1, L_{\mathrm{eff}}=L / 2$, as represented by portion A2. For $n=2$, $L_{\mathrm{eff}}=3 L / 4$, as represented by portion A3 (the beam length now taken as portion 13 for the second mode of the hinged-hinged case), and for $n=3, L_{\text {eff }}=5 L / 6$, as represented by portion A4 (the beam length now taken as portion 14 for the third mode of the hinged-hinged case).

Having seen that the guided-guided, and guided-hinged cases may be regarded as portions of a multi-span hinged-hinged beam, we now focus solely on the latter. The first spectrum of frequencies is defined by the factor $\sin \lambda_{2} L=0$, and hence $\lambda_{2} L=n \pi$, when the mode shapes are

$$
V(x)=D \sin \frac{n \pi}{L} x \quad \text { and } \quad \Psi(x)=\frac{\alpha_{2}}{(n \pi / L)} D \cos \frac{n \pi}{L} x
$$

with

$$
\alpha_{2}=\frac{\rho \omega^{2}}{\kappa G}-\left(\frac{n \pi}{L}\right)^{2}
$$

Above the cut-off frequency defined by $\omega_{\mathrm{co}}^{2}=\kappa A G / \rho I$, expression $a$, Eq. (4c), becomes negative, in which case from Eq. (4b), $\lambda_{1}^{2}$ becomes negative, and $\lambda_{1}$ becomes imaginary. This leads to the second spectrum of frequencies defined by the factor $\sinh \lambda_{1} L=0$, or i $\sin \left|\lambda_{1}\right| L=0$ and hence $\left|\lambda_{1}\right| L=n \pi$, for which the mode shapes are

$$
V(x)=\mathrm{i} B \sin \frac{n \pi}{L} x \quad \text { and } \quad \Psi(x)=\frac{\mathrm{i} \alpha_{1}}{(n \pi / L)} B \cos \frac{n \pi}{L} x .
$$

Note that the constant $B$ is assumed imaginary; moreover, the term $\alpha_{1}=\rho \omega^{2} /(\kappa G)+\lambda_{1}^{2}$ becomes equal to $\alpha_{2}$, since $\lambda_{1}=\mathrm{i}(n \pi / L)$. Thus, if one replaces $\mathrm{i} B$ by $D$ in Eqs. (11), the first spectrum mode shapes, Eqs. (10), apply equally to the second spectrum.

For both spectra, the ratio of cross-sectional rotation to shear angle, $R$, for hinged-hinged end conditions, may be determined as

$$
R=\frac{\psi}{\gamma}=\frac{\psi}{(\psi+\partial v / \partial x)}=1-\frac{\kappa G}{\rho \omega^{2}}\left(\frac{n \pi}{L}\right)^{2},
$$

which is independent of the axial coordinate. Now for a given $n$ there are two possible frequencies $\omega_{1}$ and $\omega_{2}$, so one may define

$$
R_{1}=1-\frac{\kappa G}{\rho \omega_{1}^{2}}\left(\frac{n \pi}{L}\right)^{2} \quad \text { and } \quad R_{2}=1-\frac{\kappa G}{\rho \omega_{2}^{2}}\left(\frac{n \pi}{L}\right)^{2}
$$


and since we know $\omega_{2}^{2}>\omega_{1}^{2}$, it follows that $R_{2}>R_{1}$. If one inserts numerical values into Eq. (12) from the example considered in Section 4, it is found that $R$ is negative for the first spectrum and positive for the second. More generally, if the frequency equation for the hinged-hinged case is written in the algebraic form

$$
E I\left(\frac{n \pi}{L}\right)^{4}-\rho A \omega^{2}-\rho I\left(1+\frac{E}{\kappa G}\right) \omega^{2}\left(\frac{n \pi}{L}\right)^{2}+\frac{\rho^{2} I}{\kappa G} \omega^{4}=0,
$$

then after some manipulation, one can embed $R$ into this equation to give

$$
R^{2}-\left[1-\frac{\kappa G}{E}-\left(\frac{L}{n \pi}\right)^{2} \frac{\kappa A G}{E I}\right] R-\left(\frac{L}{n \pi}\right)^{2} \frac{\kappa A G}{E I}=0
$$

this equation has the two roots $R_{1}$ and $R_{2}$ allowing one to write

$$
\left(R-R_{1}\right)\left(R-R_{2}\right)=R^{2}-\left(R_{1}+R_{2}\right) R+R_{1} R_{2}=0 .
$$

Since the coefficient of $R^{0}$ in Eq. (14) is negative (assuming $\kappa>0$ ), then by comparison with Eq. (15) one sees that the two values of $R$ must have opposing signs, irrespective of the sign of the coefficient of $R^{1}$. Thus one may conclude that $R_{1}$ is negative, and $R_{2}$ is positive; moreover $(1-R)$ must always be positive. We also note the relationship $\alpha_{2}=\left(\rho \omega^{2} / \kappa G\right) R$, so $\alpha_{2}$ is negative for the first spectrum and positive for the second.

\section{Higher-derivative Lagrangian, and the Ostrogradski and mechanical energies}

Chervyakov and Nesterenko [13,14] have shown that the fourth-order PDE describing the Timoshenko beam may be determined directly from the higher-derivative Lagrangian function

$$
L=\frac{1}{2}\left(\rho A(\dot{v})^{2}-E I\left(v^{\prime \prime}\right)^{2}-\frac{\rho^{2} I}{\kappa G}(\ddot{v})^{2}+\rho I\left(1+\frac{E}{\kappa G}\right)(\ddot{v})\left(v^{\prime \prime}\right)\right)
$$

and use of the variational principle $\delta \int_{t_{1}}^{t_{2}} \int_{0}^{L} L \mathrm{~d} x \mathrm{~d} t=0$; in the above, dot and prime denote differentiation with respect to $t$ and $x$, respectively. Performing the variation leads to Eq. (2), together with conditions at the beam ends

$$
\begin{aligned}
& \text { either } E I v^{\prime \prime \prime}-\frac{\rho I}{2}\left(1+\frac{E}{\kappa G}\right) \ddot{v}^{\prime}=0 \quad \text { or } \delta v=0, \quad v \text { is specified, } \\
& \text { either } E I v^{\prime \prime}-\frac{\rho I}{2}\left(1+\frac{E}{\kappa G}\right) \ddot{v}=0 \quad \text { or } \delta\left(v^{\prime}\right)=0, \quad v^{\prime} \text { is specified. }
\end{aligned}
$$

The physical meaning of these natural boundary conditions is not at all obvious; on the other hand, they are not inconsistent with some of the more familiar boundary conditions. For example, at a pinned end, $v$ is specified as equal to zero, in which case $\ddot{v}$ is also equal to zero, and from Eq. (18), so too is $v^{\prime \prime}$; last, from Eq. (1a), one has $\psi^{\prime}$, in which case the bending moment is zero. At a clamped end, both $v$ and $v^{\prime}$ can be specified as equal to zero; however, the alternative clamping of $v=\psi=0$ is not accommodated. At a free end, the moment and the shearing force are both zero, and again these conditions are not accommodated. 
According to the canonical formalism developed by Ostrogradski for higher-derivative theories (see Ref. [15]), the generalised coordinates $q_{1}=v$ and $q_{2}=\dot{v}$ have conjugate momenta defined by the expressions

$$
p_{2}=\frac{\partial L}{\partial(\ddot{v})}, \quad p_{1}=\frac{\partial L}{\partial(\dot{v})}-\frac{\mathrm{d}}{\mathrm{d} t}\left(\frac{\partial L}{\partial(\ddot{v})}\right),
$$

where this scheme is defined by the highest time derivative within the Lagrangian. While the physical meaning of $q_{1}$ and $q_{2}$ is clear, the momenta become

$$
p_{2}=\frac{\rho I}{2}\left(1+\frac{E}{\kappa G}\right) v^{\prime \prime}-\frac{\rho^{2} I}{\kappa G} \ddot{v}, \quad p_{1}=\rho A \dot{v}+\frac{\rho^{2} I}{\kappa G} \ddot{v}-\frac{\rho I}{2}\left(1+\frac{E}{\kappa G}\right) \dot{v}^{\prime}
$$

and their physical meaning is not at all clear. The Hamiltonian is defined by

$$
H=\int_{0}^{L}\left(p_{1} \dot{q}_{1}+p_{2} \dot{q}_{2}-L\right) \mathrm{d} x
$$

and is a conserved quantity, or an integral-invariant of the system, and is usually regarded as an energy. The values of this Hamiltonian for solutions of the relevant equations of motion have been termed the Ostrogradski energy by Nesterenko [14], and is here denoted as $E_{O}$. For the Timoshenko beam, one finds

$$
2 E_{O}=\rho A \int_{0}^{L}(\dot{v})^{2} \mathrm{~d} x+E I \int_{0}^{L}\left(v^{\prime \prime}\right)^{2} \mathrm{~d} x-\rho I\left(1+\frac{E}{\kappa G}\right) \int_{0}^{L}(\dot{v})\left(\dot{v}^{\prime \prime}\right) \mathrm{d} x+\frac{\rho^{2} I}{\kappa G} \int_{0}^{L}\left(2(\dot{v})(i \vec{v})-(i \vec{v})^{2}\right) \mathrm{d} x .
$$

For hinged-hinged end conditions, substitute

$$
v(x, t)=D \sin \frac{n \pi x}{L} \sin \omega t,
$$

which is acceptable for both spectra. We can see that $\int_{0}^{L} \sin ^{2}(n \pi x / L) \mathrm{d} x=L / 2$ for integer $n$, gives

$$
\begin{aligned}
2 E_{O}= & \frac{L D^{2}}{2} \cos ^{2} \omega t\left(\rho A \omega^{2}+\rho I\left(1+\frac{E}{\kappa G}\right) \omega^{2}\left(\frac{n \pi}{L}\right)^{2}-\frac{2 \rho^{2} I \omega^{4}}{\kappa G}\right) \\
& +\frac{L D^{2}}{2} \sin ^{2} \omega t\left(E I\left(\frac{n \pi}{L}\right)^{4}-\frac{\rho^{2} I \omega^{4}}{\kappa G}\right) .
\end{aligned}
$$

Now employ the algebraic frequency Eq. (13) to modify the first part of the right-hand side, to give

$$
2 E_{O}=\frac{L D^{2}}{2} \cos ^{2} \omega t\left(E I\left(\frac{n \pi}{L}\right)^{4}-\frac{\rho^{2} I \omega^{4}}{\kappa G}\right)+\frac{L D^{2}}{2} \sin ^{2} \omega t\left(E I\left(\frac{n \pi}{L}\right)^{4}-\frac{\rho^{2} I \omega^{4}}{\kappa G}\right)
$$

and hence

$$
E_{O}=\frac{L D^{2}}{4}\left(E I\left(\frac{n \pi}{L}\right)^{4}-\frac{\rho^{2} I \omega^{4}}{\kappa G}\right)
$$


The mechanical energy $E_{m}$ is defined by

$$
2 E_{m}=\rho A \int_{0}^{L}(\dot{v})^{2} \mathrm{~d} x+\rho I \int_{0}^{L}(\dot{\psi})^{2} \mathrm{~d} x+E I \int_{0}^{L}\left(\psi^{\prime}\right)^{2} \mathrm{~d} x+\kappa A G \int_{0}^{L}\left(v^{\prime}+\psi\right)^{2} \mathrm{~d} x .
$$

Again substitute for $v(x, t)$ as above, and

$$
\psi(x, t)=\frac{\alpha_{2}}{(n \pi / L)} D \cos \frac{n \pi x}{L} \sin \omega t \quad \text { with } \alpha_{2}=\frac{\rho \omega^{2}}{\kappa G}-\left(\frac{n \pi}{L}\right)^{2},
$$

which is valid for both spectra, and note that $\int_{0}^{L} \cos ^{2}(n \pi x / L) \mathrm{d} x=L / 2$ for integer $n$, to give the rather lengthy expression

$$
\begin{aligned}
2 E_{m}= & \frac{L D^{2}}{2} \cos ^{2} \omega t\left(\rho A \omega^{2}+\frac{\rho I \omega^{2}}{(n \pi / L)^{2}}\left\{\left(\frac{\rho \omega^{2}}{\kappa G}\right)^{2}-2\left(\frac{\rho \omega^{2}}{\kappa G}\right)\left(\frac{n \pi}{L}\right)^{2}+\left(\frac{n \pi}{L}\right)^{4}\right\}\right) \\
& +\frac{L D^{2}}{2} \sin ^{2} \omega t\left(E I\left\{\left(\frac{\rho \omega^{2}}{\kappa G}\right)^{2}-2\left(\frac{\rho \omega^{2}}{\kappa G}\right)\left(\frac{n \pi}{L}\right)^{2}+\left(\frac{n \pi}{L}\right)^{4}\right\}+\kappa A G\left(\frac{\rho \omega^{2}}{\kappa G}\right)^{2} \frac{1}{(n \pi / L)^{2}}\right) .
\end{aligned}
$$

Again by employing the algebraic frequency equation this can be simplified as

$$
E_{m}=\frac{L D^{2}}{4}\left(E I\left(\frac{n \pi}{L}\right)^{4}-\frac{\rho^{2} I \omega^{4}}{\kappa G}\right)\left[1-\frac{\left(\rho \omega^{2} / \kappa G\right)}{(n \pi / L)^{2}}\right]
$$

leading to the relationship between the two energies

$$
E_{m}=E_{O}\left[1-\frac{\left(\rho \omega^{2} / \kappa G\right)}{(n \pi / L)^{2}}\right]
$$

Nesterenko [14] has shown that the Ostrogradski energy $E_{O}$, Eq. (26), can be expressed in terms of the Euler-Bernoulli prediction for the simply supported beam, which is

$$
\omega_{\mathrm{EB}}=\left(\frac{n \pi}{L}\right)^{2} \sqrt{\frac{E I}{\rho A}}
$$

and the second spectrum cut-off frequency, $\omega_{\mathrm{co}}=\sqrt{\kappa A G / \rho I}$, as

$$
E_{O}=\frac{\rho A L D^{2}}{4}\left(\omega_{\mathrm{EB}}^{2}-\frac{\omega^{4}}{\omega_{\mathrm{co}}^{2}}\right)=\frac{\rho A L D^{2}}{4}\left(\omega_{\mathrm{EB}}-\frac{\omega^{2}}{\omega_{\mathrm{co}}}\right)\left(\omega_{\mathrm{EB}}+\frac{\omega^{2}}{\omega_{\mathrm{co}}}\right)
$$

he then employed an asymptotic expansion, valid for a long beam, to show that $E_{O}$ is positive for the first spectrum of frequencies, and negative for the second. One might suspect this to be the case from the following arguments: the factor $\left(\omega_{\mathrm{EB}}+\omega^{2} / \omega_{\mathrm{co}}\right)$ is always positive. For the first spectrum, the Timoshenko frequency prediction $\omega_{1}$ is always smaller than the Euler-Bernoulli prediction, so the factor $\left(\omega_{\mathrm{EB}}-\omega^{2} / \omega_{\mathrm{co}}\right)$ is positive at least over the range, where $\omega_{1}$ is smaller than the cut-off frequency. For the second spectrum, the Timoshenko frequency prediction $\omega_{2}$ is 
greater than the cut-off frequency, so this factor is negative at least when $\omega_{2}$ is greater than the Euler-Bernoulli prediction.

A more rigorous approach is to note that the algebraic frequency equation must factorise as $\left(\omega^{2}-\omega_{1}^{2}\right)\left(\omega^{2}-\omega_{2}^{2}\right)=0$, so one has $\omega^{4}-\left(\omega_{1}^{2}+\omega_{2}^{2}\right) \omega^{2}+\omega_{1}^{2} \omega_{2}^{2}=0$; now multiply this throughout by the term $\rho^{2} I / \kappa G$, and comparing with Eq. (13), one must have

$$
E I\left(\frac{n \pi}{L}\right)^{4}=\frac{\rho^{2} I}{\kappa G} \omega_{1}^{2} \omega_{2}^{2} .
$$

The Ostrogradski energy, Eq. (26), becomes

$$
E_{O}=\frac{L D^{2} \rho^{2} I}{4 \kappa G}\left(\omega_{1}^{2} \omega_{2}^{2}-\omega^{4}\right) .
$$

Now for a given $n$, we know that $\omega_{2}^{2}>\omega_{1}^{2}$; for the first spectrum one may write

$$
E_{O 1}=\frac{L D_{1}^{2} \rho^{2} I}{4 \kappa G}\left(\omega_{1}^{2} \omega_{2}^{2}-\omega_{1}^{4}\right)=\frac{L D_{1}^{2} \rho^{2} I}{4 \kappa G} \omega_{1}^{2}\left(\omega_{2}^{2}-\omega_{1}^{2}\right)>0,
$$

while for the second

$$
E_{O 2}=\frac{L D_{2}^{2} \rho^{2} I}{4 \kappa G}\left(\omega_{1}^{2} \omega_{2}^{2}-\omega_{2}^{4}\right)=\frac{L D_{2}^{2} \rho^{2} I}{4 \kappa G} \omega_{2}^{2}\left(\omega_{1}^{2}-\omega_{2}^{2}\right)<0 .
$$

Thus one sees quite unequivocally that $E_{O}$ is positive for the first spectrum and negative for the second. If both modes are present simultaneously, then adding gives

$$
E_{O}=\frac{L \rho^{2} I}{4 \kappa G}\left(\omega_{2}^{2}-\omega_{1}^{2}\right)\left(D_{1}^{2} \omega_{1}^{2}-D_{2}^{2} \omega_{2}^{2}\right),
$$

a result presented previously by Chervyakov and Nesterenko [13].

An alternative approach focuses on the factor relating $E_{O}$ and the mechanical energy, $E_{m}$, in Eq. (31), noting that $E_{m}$ is always positive. For both spectra, the ratio of cross-sectional rotation to shear angle, $R$, for hinged-hinged end conditions, is determined as

$$
R=\frac{\psi}{\gamma}=\frac{\psi}{(\psi+\partial v / \partial x)}=1-\frac{\kappa G}{\rho \omega^{2}}\left(\frac{n \pi}{L}\right)^{2},
$$

allowing one to re-write Eq. (31) as

$$
E_{m}=E_{O} \frac{R}{R-1}
$$

or equivalently

$$
E_{m}=E_{O} \frac{\psi}{\psi-\gamma}=\frac{\psi}{-(\partial v / \partial x)} .
$$

Now $R$ is negative for the first spectrum (using the above sign conventions), and positive for the second; it is also always smaller than unity for $n \neq 0$. From Eq. $(37),(1-R)$ is always positive. Thus again one concludes that $E_{O}$ is positive for the first spectrum, and negative for the second. Although the physical significance of the Ostrogradski energy remains uncertain, it provides a clear indication of a character difference between the two spectra. 
An example of an uncontroversial higher time derivative theory is the coupled tension-torsion coupling of pre-twisted structures such as a turbine blade; the static behaviour is assumed to be governed by coupled equations of the form

$$
\left[\begin{array}{c}
T_{x} \\
M_{x}
\end{array}\right]=\left[\begin{array}{ll}
E A & K_{t t} \\
K_{t t} & G J
\end{array}\right]\left[\begin{array}{l}
\frac{\partial u}{\partial x} \\
\frac{\partial \theta}{\partial x}
\end{array}\right]
$$

and the dynamic equations are

$$
\left[\begin{array}{ll}
E A & K_{t t} \\
K_{t t} & G J
\end{array}\right]\left[\begin{array}{l}
\frac{\partial^{2} u}{\partial x^{2}} \\
\frac{\partial^{2} \theta}{\partial x^{2}}
\end{array}\right]=\left[\begin{array}{c}
\rho A \frac{\partial^{2} u}{\partial t^{2}} \\
J_{x} \frac{\partial^{2} \theta}{\partial t^{2}}
\end{array}\right] .
$$

Note the symmetry of the stiffness matrix. For the straight beam there would be zero coupling, that is $K_{t t}=0$, and the above reduces to two uncoupled equations of the second order

$$
E A \frac{\partial^{2} u}{\partial x^{2}}=\rho A \frac{\partial^{2} u}{\partial t^{2}} \text { and } G J \frac{\partial^{2} \theta}{\partial x^{2}}=J_{x} \frac{\partial^{2} \theta}{\partial t^{2}} .
$$

Elimination of one of the variables, say $u$, in favour of $\theta$, would lead to a fourth-order equation in both time and space, as

$$
\left(E A G J-K_{t t}^{2}\right) \frac{\partial^{4} \theta}{\partial x^{4}}-\left(E A J_{x}+\rho A G J\right) \frac{\partial^{4} \theta}{\partial x^{2} \partial t^{2}}+\rho A J_{x} \frac{\partial^{4} \theta}{\partial t^{4}}=0 .
$$

Eliminating $\theta$ in favour of $u$ would give an equivalent equation; assuming sinusoidal time dependence leads to

$$
\left(E A G J-K_{t t}^{2}\right) \frac{\mathrm{d}^{4} \Theta}{\mathrm{d} x^{4}}+\omega^{2}\left(G J \rho A+E A J_{x}\right) \frac{\mathrm{d}^{2} \Theta}{\mathrm{d} x^{2}}+\omega^{4} \rho A J_{x} \Theta=0 .
$$

Typically, uncoupled extensional frequencies are larger than torsional, for the same wavelength. Coupling has the effect of bringing these close together - the torsional frequency is raised, and the extensional is lowered, although not by so much; the higher spectrum is predominantly extensional with a small amount of torsion, the lower is predominantly torsion with a small amount of extension. For (co)sinusoidal mode shapes (free-free and fixed-fixed end conditions), the natural frequency predictions are

predominantly torsional

$$
\omega=\frac{n \pi}{L} \sqrt{\frac{E A J_{x}+G J \rho A-\sqrt{\left(E A J_{x}-G J \rho A\right)^{2}+4 \rho A J_{x} K_{t t}^{2}}}{2 \rho A J_{x}}},
$$

predominantly extensional

$$
\omega=\frac{n \pi}{L} \sqrt{\frac{E A J_{x}+G J \rho A+\sqrt{\left(E A J_{x}-G J \rho A\right)^{2}+4 \rho A J_{x} K_{t t}^{2}}}{2 \rho A J_{x}}}
$$


and setting $K_{t t}=0$, leads to familiar expressions for the two established uncoupled theories; this contrasts with "uncoupling" of the Timoshenko equations, as described above, when one branch tends to the Euler-Bernoulli frequency prediction, the other to infinity. Moreover, one can show that the Ostrogradski energy is positive for both modes.

\section{Numerical example}

We now extend the calculations presented by Levinson and Cooke [4]; the beam has length $L=0.5 \mathrm{~m}$, depth $h=0.125 \mathrm{~m}$, Young's modulus $E=210 \times 10^{9} \mathrm{~N} / \mathrm{m}^{2}$, density $\rho=7850 \mathrm{~kg} / \mathrm{m}^{3}$, Poisson's ratio $v=0.3$, and unit thickness consistent with plane stress conditions. First, note that the Euler-Bernoulli prediction for the fundamental frequency $(n=1)$ is $\omega_{1}=7368 \mathrm{rad} / \mathrm{s}$ which is some $9.5 \%$ in excess of the exact value, so for this short beam a Timoshenko model should always be used. Two values of the shear coefficient are employed in the comparison: $\kappa=\frac{5}{6}$ as in [4], and $\kappa=5(1+v) /(6+5 v)$. The exact plane stress theory is presented in Appendix A.

From Table 1, one concludes initially what has been reported several times over-TBT1 can provide excellent agreement with exact theory when one uses the best shear coefficient $\kappa=5(1+v) /(6+5 v)$, as in column 5 ; the error is better than -0.4 and $+0.55 \%$. On the other hand, the value $\kappa=\frac{5}{6}$ provides a prediction always lower than the exact, at least over the range considered, with a maximum error of $-2 \%$. Experimental evidence in support of the value $\kappa=5(1+v) /(6+5 v)$ has recently been provided by Méndez-Sánchez et al. [17], although they also note that a two-coefficient theory presented by Stephen and Levinson [18], and which incorporates both this, and Cowper's value [19] of $\kappa=10(1+v) /(12+11 v)$, provides marginally better agreement with their experimental results.

The behaviour of both the first and higher spectra of the (a)symmetric cases is shown in Fig. 2; first note that the differences between TBT1 and the lowest exact asymmetric (flexural) prediction, denoted A1, are not discernable on this scale. The second spectrum prediction TBT2, is very close to the second exact asymmetric prediction (A2) for $n \leqslant 2$, which is consistent with Miklowitz [11]. However, TBT2 is closest to the second symmetric prediction (S2) for $3 \leqslant n \leqslant 8$, and closest to the third asymmetric (A3) for $9 \leqslant n \leqslant 11$. This capricious behaviour is the same as was found in Ref. [2] for the beam of circular cross-section, and for the $w_{2}$-mode of Mindlin plate theory [3] - at various wavelengths (equivalently mode numbers), it agrees perfectly with exact theory, but never the same mode! Also note that the lowest symmetric (extensional) frequency (S1) and the lowest asymmetric (flexural) frequency (A1) converge as the wavelength becomes small (equivalently $n$ becomes large); one would expect as much, as both cases should predict wave propagation at Rayleigh surface velocity at very small wavelength. From a standing wave viewpoint, the data shown in Fig. 2 are strictly only true for integer $n$. However, the figure can also be interpreted as a dispersion diagram for wave propagation in an infinite beam when the wavelength is $\lambda=2 L / n$.

In passing, it is instructive to compare the exact extensional frequency predictions, with those of the classic theory, and that of Rayleigh-Love, which includes a Poisson's ratio correction, Table 2. It is clear that the Rayleigh-Love theory is better than the classic, but it cannot rival the level of accuracy that TBT1 provides for the asymmetric case. 
Table 1

Lowest exact asymmetric, and first TBT spectrum frequency predictions

\begin{tabular}{rrrrc}
\hline$n$ & Exact-asymmetric & $\kappa=\frac{5}{6}$ & $\kappa=5(1+v) /(6+5 v)$ & Percentage error \\
\hline 1 & 6730 & 6712 & 6729 & -0.015 \\
2 & 2296 & 22136 & 22279 & -0.076 \\
3 & 41166 & 40701 & 41094 & -0.175 \\
4 & 61051 & 60170 & 60890 & -0.264 \\
5 & 81163 & 79806 & 80896 & -0.329 \\
6 & 101226 & 99375 & 100856 & -0.366 \\
7 & 121149 & 118812 & 120693 & -0.376 \\
8 & 140907 & 138112 & 140396 & -0.363 \\
9 & 160503 & 157287 & 159974 & -0.330 \\
10 & 179948 & 176355 & 179443 & -0.281 \\
11 & 199253 & 195333 & 198820 & -0.217 \\
12 & 218433 & 214236 & 218118 & -0.144 \\
13 & 237501 & 233077 & 237351 & -0.063 \\
14 & 256469 & 251864 & 256529 & +0.023 \\
15 & 275350 & 270608 & 275660 & +0.113 \\
16 & 294156 & 289314 & 294752 & +0.203 \\
17 & 312898 & 307987 & 313810 & +0.291 \\
18 & 331585 & 326634 & 332838 & +0.378 \\
19 & 350227 & 345256 & 351842 & +0.461 \\
20 & 368833 & 363859 & 370823 & +0.540 \\
\hline
\end{tabular}

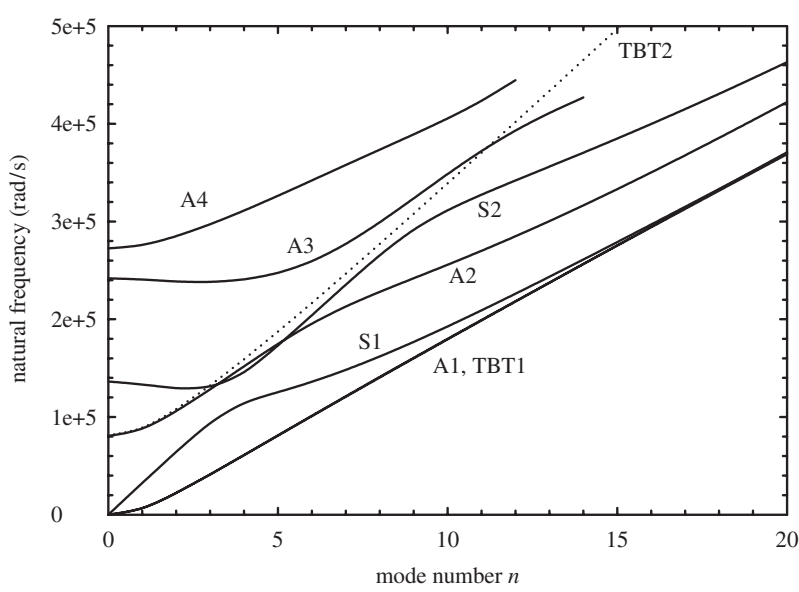

Fig. 2. Natural frequency predictions for a hinged-hinged beam of narrow rectangular cross-section for a range of mode numbers, $n$. TBT1 and TBT2 denote the first and second spectrum predictions, respectively, of Timoshenko beam theory. A and S are the asymmetric (flexural) and symmetric (extensional) predictions, respectively, from plane stress elasticity theory; numerals indicate first, second, etc. The difference between TBT1 and A1 is not discernable.

\section{Conclusions}

Recent contributions to the debate on the second frequency spectrum of Timoshenko beam theory have been reviewed, and several new results are presented. In particular, it is shown that 
Table 2

Comparison of extensional (symmetric) frequency predictions

\begin{tabular}{lccc}
\hline$n$ & Exact-symmetric & Classic & Rayleigh-Love \\
\hline 1 & 32418 & 32498 & 32423 \\
2 & 64214 & 64996 & 64403 \\
3 & 93518 & 97494 & 95525 \\
4 & 114010 & 129991 & 125432 \\
\hline
\end{tabular}

the frequency equation factorises not only for hinged-hinged end conditions, as is often claimed, but also for guided-guided and guided-hinged end conditions. These new beam end combinations may be regarded as portions of a multi-span hinged-hinged beam.

A higher-derivative Lagrangian which, through Hamilton's principle, leads directly to the wellknown fourth-order Timoshenko beam equation is also reviewed; the meaning of the natural boundary conditions leading from the variational process is not clear, and can be reconciled with just some of the familiar beam end conditions. Likewise the canonical momenta associated with the generalised coordinates do not have any obvious physical meaning. A simple relationship between the so-called Ostrogradski energy and the mechanical energy is derived for hinged-hinged end conditions. Both are integral-invariants of the system. While the latter is positive definite, it is shown that the Ostrogradski energy is positive for the first spectrum but negative for the second; within some branches of physics, this would be sufficient evidence to conclude that the second spectrum in "unphysical".

The numerical example of a short hinged-hinged beam of thin rectangular cross-section considered by Levinson and Cooke is re-examined. It is seen that agreement between the Timoshenko first spectrum frequency prediction and exact plane stress elastodynamic theory is excellent. However, the Timoshenko second spectrum prediction does not provide consistent agreement with any single mode. Again it is concluded that these second spectrum predictions should be disregarded.

\section{Appendix A. Plane stress elastodynamic theory}

The plane stress equivalent of the plane strain Rayleigh-Lamb theory of plate flexural vibration was developed first by Timoshenko [20] and more recently by Cowper [21]. Here, the theory is extended to include both asymmetric (flexural) and symmetric (extensional) cases. Cut-off frequencies are also included. The governing equations are

$$
\frac{\partial \sigma_{x}}{\partial x}+\frac{\partial \tau_{x y}}{\partial y}+\rho \omega^{2} u=0, \quad \frac{\partial \tau_{x y}}{\partial x}+\frac{\partial \sigma_{y}}{\partial y}+\rho \omega^{2} v=0,
$$

together with Hooke's law

$$
\left.\sigma_{x}=\frac{E}{\left(1-v^{2}\right)}\left(\varepsilon_{x}+v \varepsilon_{y}\right), \quad \sigma_{y}=\frac{E}{\left(1-v^{2}\right)}\left(\varepsilon_{y}+v \varepsilon_{x}\right), \quad \tau_{x y}=\frac{E}{2(1+v)} \gamma_{x y}=G \gamma_{x y} . \quad \text { (A. } 2 \mathrm{a}-\mathrm{c}\right)
$$


These are subject to traction-free boundary conditions on the upper and lower edge of the strip $y= \pm h / 2$.

\section{A.1. Asymmetric case}

The displacement field

$$
\begin{aligned}
& u=\left(A \sinh \frac{\alpha y}{h}+B \sinh \frac{\beta y}{h}\right) \cos \frac{n \pi x}{L} \sin \omega t, \\
& v=\left(C \cosh \frac{\alpha y}{h}+D \cosh \frac{\beta y}{h}\right) \sin \frac{n \pi x}{L} \sin \omega t
\end{aligned}
$$

satisfies the conditions of a simple-support, as both $v$ and $\sigma_{x}$ are equal to zero on $x=0$ and $x=L$ for integer $n$. In order that the dynamic equilibrium Eqs. (A.1a,b) are satisfied, one requires

$$
C=\left(\frac{n \pi h}{\alpha L}\right) A \quad \text { and } \quad D=\left(\frac{\beta L}{n \pi h}\right) B
$$

and the relationships

$$
\alpha^{2}=\left(\frac{n \pi h}{L}\right)^{2}-\frac{\rho \omega^{2} h^{2}}{G}, \quad \beta^{2}=\left(\frac{n \pi h}{L}\right)^{2}-\frac{\rho \omega^{2} h^{2}\left(1-v^{2}\right)}{E} .
$$

Traction-free boundary conditions on the lower and upper edges are $\sigma_{y}=\tau_{x y}=0$ on $y= \pm h / 2$, leading to the matrix equation

$$
\left[\begin{array}{cc}
(1-v) \sinh \frac{\alpha}{2} & \left(\left(\frac{\beta L}{n \pi h}\right)^{2}-v\right) \sinh \frac{\beta}{2} \\
\left(\left(\frac{n \pi}{L}\right)^{2} \frac{h}{\alpha}+\frac{\alpha}{h}\right) \cosh \frac{\alpha}{2} & \frac{2 \beta}{h} \cosh \frac{\beta}{2}
\end{array}\right]\left[\begin{array}{l}
A \\
B
\end{array}\right]=\mathbf{0}
$$

and for a non-trivial solution, one requires that the determinant should be zero. Note the error in Eq. (4b) of Cowper [21], which has a minus sign in the element below the leading diagonal.

The asymmetric cut-off frequency is defined by the special case of $n=0$, when the displacement component $v$, and both the direct strains $\varepsilon_{x}$ and $\varepsilon_{y}$, and hence the direct stress components, are equal to zero; thus the stress field is free of dilatation, and involves shear only. The shearing stress $\tau_{x y}$ is independent of $x$, so the dynamic equilibrium Eq. (A.1b) is satisfied identically. Equilibrium Eq. (A.1a) becomes

$$
A \sinh \frac{\alpha y}{h}\left(\frac{G \alpha^{2}}{h^{2}}+\rho \omega_{\mathrm{co}}^{2}\right)+B \sinh \frac{\beta y}{h}\left(\frac{G \beta^{2}}{h^{2}}+\rho \omega_{\mathrm{co}}^{2}\right)=0
$$

and is satisfied if one sets $A=B, \alpha=\beta$, when a non-trivial solution requires $\alpha^{2}=-\rho \omega_{\mathrm{co}}^{2} h^{2} / G$. The traction-free requirement $\tau_{x y}=0$ on $y= \pm h / 2$ leads to the requirement $\cosh (\alpha / 2)=$ $\cos (\mathrm{i} \alpha / 2)=0$, hence $\rho \omega_{\mathrm{co}}^{2} h^{2} / G=\pi^{2}, 9 \pi^{2}, 25 \pi^{2}, \ldots$, etc, and radian frequencies

$$
\omega_{\mathrm{co}}=(2 m-1) \frac{\pi}{h} \sqrt{\frac{G}{\rho}}, \quad m=1,2,3, \ldots, \text { etc. }
$$




\section{A.2. Symmetric case}

The displacement field

$$
\begin{aligned}
& u=\left(A \cosh \frac{\alpha y}{h}+B \cosh \frac{\beta y}{h}\right) \sin \frac{n \pi x}{L} \sin \omega t, \\
& v=\left(C \sinh \frac{\alpha y}{h}+D \sinh \frac{\beta y}{h}\right) \cos \frac{n \pi x}{L} \sin \omega t
\end{aligned}
$$

now requires

$$
C=-\left(\frac{n \pi h}{\alpha L}\right) A \quad \text { and } \quad D=-\left(\frac{\beta L}{n \pi h}\right) B
$$

with $\alpha$ and $\beta$ as defined previously. Displacement $u$ is now equal to zero on $x=0$ and $x=L$ for integer $n$; stress $\sigma_{x}$ and displacement $v$ are non-zero on these ends, so this field is consistent with a fixed end that allows Poisson's ratio contraction effects. Again traction-free conditions on the lower and upper edges lead to the matrix equation

$$
\left[\begin{array}{cc}
(1-v) \cosh \frac{\alpha}{2} & \left(\left(\frac{\beta L}{n \pi h}\right)^{2}-v\right) \cosh \frac{\beta}{2} \\
\left(\left(\frac{n \pi}{L}\right)^{2} \frac{h}{\alpha}+\frac{\alpha}{h}\right) \sinh \frac{\alpha}{2} & \frac{2 \beta}{h} \sinh \frac{\beta}{2}
\end{array}\right]\left[\begin{array}{l}
A \\
B
\end{array}\right]=\mathbf{0} ;
$$

note that this is identical in form to the asymmetric case, except that the sinh and cosh functions are interchanged. Again, a non-trivial solution requires the determinant to be zero.

The symmetric cut-off frequency is defined by the special case of $n=0$, when the displacement component $u$ is now zero, and $v$ is independent of $x$. Immediately, the shearing stress $\tau_{x y}$ is zero. The dynamic equilibrium Eq. (A.1a) is satisfied identically, while Eq. (A.1b) yields

$$
C \sinh \frac{\alpha y}{h}\left(\frac{E \alpha^{2}}{\left(1-v^{2}\right) h^{2}}+\rho \omega_{\mathrm{co}}^{2}\right)+D \sinh \frac{\beta y}{h}\left(\frac{E \beta^{2}}{\left(1-v^{2}\right) h^{2}}+\rho \omega_{\mathrm{co}}^{2}\right)=0
$$

and is satisfied if one sets $C=D, \alpha=\beta$, when a non-trivial solution requires $\alpha^{2}=$ $-\left(1-v^{2}\right) \rho \omega_{\mathrm{co}}^{2} h^{2} / E$. The traction-free requirement $\sigma_{y}=0$ on $y= \pm h / 2$ again leads to the requirement $\cosh (\alpha / 2)=\cos (\mathrm{i} \alpha / 2)=0$, hence $\left(1-v^{2}\right) \rho \omega_{\mathrm{co}}^{2} h^{2} / E=\pi^{2}, 9 \pi^{2}, 25 \pi^{2}, \ldots$, etc., and radian frequencies

$$
\omega_{\mathrm{co}}=(2 m-1) \frac{\pi}{h} \sqrt{\frac{E}{\left(1-v^{2}\right) \rho}}, \quad m=1,2,3, \ldots \text { etc. }
$$

\section{References}

[1] R.W. Traill-Nash, A.R. Collar, The effect of shear flexibility and rotary inertia on the bending vibrations of beams, Quarterly Journal of Mechanics and Applied Mathematics 6 (1953) 186-222. 
[2] N.G. Stephen, The second frequency spectrum of Timoshenko beams, Journal of Sound and Vibration 80 (1982) 578-582.

[3] N.G. Stephen, Mindlin plate theory-best shear coefficient and higher spectra validity, Journal of Sound and Vibration 202 (1997) 539-553.

[4] M. Levinson, D.W. Cooke, On the two frequency spectra of Timoshenko beams, Journal of Sound and Vibration 84 (1982) 319-326.

[5] G. Prathap, The two frequency spectra of Timoshenko beams - a re-assessment, Journal of Sound and Vibration 90 (1983) 443-445 See also M. Levinson AUTHOR'S REPLY 445-446.

[6] G.R. Bhashyam, G. Prathap, The second frequency spectrum of Timoshenko beams, Journal of Sound and Vibration 76 (1981) 407-420.

[7] B.A.H. Abbas, J. Thomas, The second frequency spectrum of Timoshenko beams, Journal of Sound and Vibration 51 (1977) 123-137.

[8] S.M. Han, H. Benaroya, T. Wei, Dynamics of transversely vibrating beams using four engineering theories, Journal of Sound and Vibration 225 (1999) 935-988.

[9] B. Downs, Vibration of a uniform, simply supported Timoshenko beam without transverse deflection, $A S M E$ Journal of Applied Mechanics 43 (1976) 671-674.

[10] S. Ekwaro-Osire, D.H.S. Maithripala, J.M. Berg, A series expansion approach to interpreting the spectra of the Timoshenko beam, Journal of Sound and Vibration 240 (2001) 667-678.

[11] J. Miklowitz, The Theory of Elastic Waves and Waveguides, North-Holland, Amsterdam, 1978 (See Section 7.1.2.2, p. 381).

[12] X.Q. Wang, R.M.C. So, Various standing waves in a Timoshenko beam, Journal of Sound and Vibration 280 (2005) 311-328.

[13] A.M. Chervyakov, V.V. Nesterenko, Is it possible to assign physical meaning to field theory with higher derivatives?, Physical Review D 48 (1993) 5811-5817.

[14] V.V. Nesterenko, A theory for transverse vibrations of a Timoshenko beam, PMM Journal of Applied Mathematics and Mechanics 57 (1993) 669-677.

[15] E.T. Whittaker, A Treatise on the Analytical Dynamics of Particles and Rigid Bodies, fourth ed., Cambridge University Press, Cambridge, 1937 pp. 265-267.

[16] J.Z. Simon, Higher derivative Lagrangians, nonlocality, problems and solutions, Physical Review D 41 (1990) $3720-3733$.

[17] R.A. Méndez-Sánchez, A. Morales, J. Flores, Experimental check on the accuracy of Timoshenko's beam theory, Journal of Sound and Vibration 279 (2005) 508-512.

[18] N.G. Stephen, M. Levinson, A second order beam theory, Journal of Sound and Vibration 67 (1979) 293-305.

[19] G.R. Cowper, The shear coefficient in Timoshenko's beam theory, ASME Journal of Applied Mechanics 33 (1966) $335-340$

[20] S.P. Timoshenko, On the transverse vibrations of bars of uniform cross-section, Philosophical Magazine 43 (1922) $125-131$.

[21] G.R. Cowper, On the accuracy of Timoshenko's beam theory, Proceedings of the ASCE Journal of the Engineering Mechanics Division 94 (1968) 1447-1453. 